\title{
THE NON-EMPTINESS OF JOINT SPECTRAL SUBSETS OF EUCLIDEAN $n$-SPACE
}

\author{
W. J. RICKER and A. R. SCHEP
}

(Received 1 April 1988)

Communicated by $\mathbf{R}$. O. Vyborny

\begin{abstract}
A. McIntosh and A. Pryde introduced and gave some applications of a notion of "spectral set", $\gamma(\mathbf{T})$, associated with each finite, commuting family of continuous linear operators $\mathbf{T}$ in a Banach space. Unlike most concepts of joint spectrum, the set $\gamma(\mathbf{T})$ is part of real Euclidean space. It is shown that $\gamma(\mathbf{T})$ is always non-empty whenver there are at least two operators in $\mathbf{T}$.
\end{abstract}

1980 Mathematics subject classification (Amer. Math. Soc.) (1985 Revision): 47 A 10.

Let $X$ be a complex Banach space and $\mathrm{T}=\left(T_{1}, \ldots, T_{n}\right)$ be a commuting $n$ tuple of continuous linear operators in $X$; the space of all such operators in $X$ is denoted by $L(X)$. A joint spectral set $\gamma(\mathbf{T}) \subset \mathbf{R}^{n}$ is defined by

(1) $\gamma(\mathbf{T})=\left\{\left(u_{1}, \ldots, u_{n}\right) \in \mathbf{R}^{n}: \sum_{j=1}^{n}\left(T_{j}-u_{j} I\right)^{2}\right.$ is not invertible in $\left.L(X)\right\}$,

where $I$ is the identity operator in $X$ and $\mathbf{R}$ denotes the real numbers [1]. This notion has proved to be useful in determining functional calculi for certain $n$-tuples $\mathbf{T}$ with applications to finding estimates for the solution of linear systems of operator equations [1, 2, 3]. For applications to other notions of joint spectra we refer to [4].

It is known that $\gamma(\mathbf{T})$ is always a compact subset of $\mathbf{R}^{n}$ [3, Theorem 4.1]. The question arises of whether or not $\gamma(\mathbf{T})$ is empty? If $n=1$, then it is easy

Both authors gratefully acknowledge the support of an Alexander von Humboldt Fellowship while visiting the Federal Republic of Germany.

(c) 1989 Australian Mathematical Society 0263-6115/89 \$A2.00+0.00 
to check that $\gamma(\mathbf{T})=\sigma(\mathbf{T}) \cap \mathbf{R}$ and so $\gamma(\mathbf{T})$ may be empty in this case. For $n \geq 2$, it is known that $\gamma(\mathbf{T}) \neq \varnothing$ if each operator $T_{j}, 1 \leq j \leq n$, has real spectrum. Indeed, in this case $\gamma(\mathbf{T})$ coincides with the joint Taylor spectrum of $\mathbf{T}$ [4, Theorem 1] and so is certainly non-empty. By a judicious use of Clifford analysis and monogenic functions, McIntosh and Pryde have shown that $\gamma(\mathbf{T}) \neq \varnothing$ for arbitrary commuting $n$-tuples $\mathbf{T}$ whenever $n$ is an even integer [3, Section 3]. If the underlying Banach space $X$ is finite dimensional, then it is shown in [5] that $\gamma(\mathbf{T}) \neq \varnothing$ for arbitrary commuting $n$-tuples $\mathbf{T}$ such that $n \geq 2$. In this note we show that $\gamma(\mathbf{T})$ is always non-empty whenever $n \geq 2$; there are no restrictions on $\mathbf{T}$ or on the Banach space $X$. Let us record this statement formally.

Theorem 1. Let $X$ be a Banach space, $n \geq 2$ be an integer and $\mathrm{T}=$ $\left(T_{1}, \ldots, T_{n}\right)$ be a commuting $n$-tuple of elements from $L(X)$. Then $\gamma(\mathbf{T})$ is non-empty.

The proof is based on some elementary Banach algebra theory combined with an analogue of the computation given in the proof of [5, Theorem 1].

Let $\mathfrak{A}(\mathbf{T})=\{\mathbf{T}\}^{c c}$ denote the bicommutant of $\left\{T_{j}: 1 \leq j \leq n\right\}$ in $L(X)$. Then $\mathfrak{A}(\mathbf{T})$ is a closed, abelian Banach subalgebra of $L(X)$ containing the identity operator $I$. In addition, $\mathfrak{A}(\mathrm{T})$ is inverse closed in $L(X)$. That is, if $S \in \mathfrak{A}(\mathbf{T})$ is invertible in $L(X)$, then $S^{-1} \in \mathfrak{A}(\mathbf{T})$. This is a consequence of the identity $\mathfrak{A}(\mathbf{T})^{c c}=\mathfrak{A}(\mathbf{T})$ and the fact that $S^{-1} \in\{S\}^{c c}$ whenever $S \in L(X)$ is invertible. Of course, if $S \in \mathfrak{A}(\mathbf{T})$, then $\{S\}^{c c} \subseteq \mathfrak{A}(\mathbf{T})^{c c}$. It follows that

$$
\sigma_{\mathfrak{A}(\mathbf{T})}(S)=\sigma_{L(X)}(S), \quad S \in \mathfrak{A}(\mathbf{T}),
$$

where $\sigma_{\mathfrak{a}(\mathbf{T})}$ and $\sigma_{L(X)}$ denote the spectrum relative to the Banach algebra $\mathfrak{A}(\mathbf{T})$ and $L(X)$, respectively. It is clear from (2) and the definition of $\gamma(\mathbf{T})$ that

$$
\gamma(\mathbf{T})=\left\{\left(u_{1}, \ldots, u_{n}\right) \in \mathbf{R}^{n}: 0 \in \sigma_{\mathfrak{A}(\mathbf{T})}\left(\sum_{j=1}^{n}\left(T_{j}-u_{j} I\right)^{2}\right)\right\} .
$$

Furthermore, since $\sum_{j=1}^{n}\left(T_{j}-u_{j} I\right)^{2}$ actually belongs to $\mathfrak{A}(\mathrm{T})$, for every $\mathbf{u}=$ $\left(u_{1}, \ldots, u_{n}\right)$ in $\mathbf{R}^{n}$, it suffices to show that

$$
\gamma(\mathbf{b})=\left\{\mathbf{u} \in \mathbf{R}^{n} ; 0 \in \sigma_{\mathfrak{B}}\left(\sum_{j=1}^{n}\left(b_{j}-u_{j} e\right)^{2}\right)\right\}
$$

is non-empty, whenever $\mathfrak{B}$ is a commutative Banach algebra (with unit $e$ ), $n \geq 2$ is an integer and $\mathbf{b}=\left(b_{1}, \ldots, b_{n}\right) \in \mathfrak{B}^{n}$.

To establish this we proceed as follows. Let $\mathfrak{M}$ be the maximal ideal space of $\mathfrak{B}$. It follows from standard Banach algebra theory that $\mathfrak{B}$ can be 
identified with a subalgebra $\hat{\mathfrak{B}}$ of the space $C(\mathfrak{M})$ of continuous functions on $\mathfrak{M}$. The Gelfand transform $\hat{\imath}: \mathfrak{B} \rightarrow \hat{\mathfrak{B}}$ is a homomorphism such that $\hat{e}=1$ (the constant function 1 on $\mathfrak{M}$ ) and

$$
\sigma_{\mathfrak{B}}(b)=\hat{b}(\mathfrak{M})=\{\hat{b}(m) ; m \in \mathfrak{M}\}, \quad b \in \mathfrak{B} .
$$

The homomorphism property of the Gelfand transform implies that

$$
\left[\sum_{j=1}^{n}\left(b_{j}-u_{j} e\right)^{2}\right]^{-}=\sum_{j=1}^{n}\left(\hat{b}_{j}-u_{j} 1\right)^{2}, \quad \mathbf{u} \in \mathbf{R}^{n}
$$

for every $\mathbf{b} \in \mathfrak{B}^{n}$. This identity, together with (4), implies that

$$
0 \in \sigma_{\mathfrak{B}}\left(\sum_{j=1}^{n}\left(b_{j}-u_{j} e\right)^{2}\right) \quad \text { if and only if } \sum_{j=1}^{n}\left(\hat{b}_{j}(m)-u_{j}\right)^{2}=0,
$$

for some $m \in \mathfrak{M}$. It follows immediately from (3) that

$$
\gamma(\mathbf{b})=\bigcup_{m \in \mathfrak{M}}\left\{\mathbf{u} \in \mathbf{R}^{n}: \sum_{j=1}^{n}\left(\hat{b}_{j}(m)-u_{j}\right)^{2}=0\right\} .
$$

So, to show $\gamma(\mathbf{b})$ is non-empty it suffices to show that there exists $m \in \mathfrak{M}$ for which the set

$$
Z(\hat{\mathbf{b}}, m)=\left\{\mathbf{u} \in \mathbf{R}^{n}: \sum_{j=1}^{n}\left(\hat{b}_{j}(m)-u_{j}\right)^{2}=0\right\}
$$

is non-empty. Actually, we will show that $Z(\hat{\mathbf{b}}, m) \neq \varnothing$ for every $m \in \mathfrak{M}$. So, fix $m \in \mathfrak{M}$. Write $\hat{b}_{j}(m)=a_{j}(m)+i c_{j}(m), 1 \leq j \leq n$, with $a_{j}(m)$ and $c_{j}(m)$ being real numbers. Then $\mathbf{u} \in \mathbf{R}^{n}$ satisfies $\sum_{j=1}^{n}\left(\hat{b}_{j}(m)-u_{j}\right)^{2}=0$ if and only if

$$
\sum_{j=1}^{n}\left(u_{j}-a_{j}(m)\right)^{2}=\sum_{j=1}^{n} c_{j}(m)^{2}
$$

and simultaneously

$$
\sum_{j=1}^{n}\left(u_{j}-a_{j}(m)\right) c_{j}(m)=0
$$

Considering $\mathbf{u} \in \mathbf{R}^{n}$ as a variable, (7.1) is the equation of a sphere in $\mathbf{R}^{n}$ centred at $\mathbf{a}(m)=\left(a_{1}(m), \ldots, a_{n}(m)\right)$ and with radius

$$
\|\mathbf{c}(m)\|_{2}=\left(\sum_{j=1}^{n} c_{j}(m)^{2}\right)^{1 / 2}
$$


and (7.2) is a hyperplane in $\mathbf{R}^{n}$ with normal $\mathbf{c}(m)$ and passing through $\mathbf{a}(m)$. So, if $n \geq 2$, then there certainly are simultaneous solutions of (7.1) and (7.2) and hence, $Z(\hat{\mathbf{b}}, m) \neq \varnothing$ (for every $m \in \mathfrak{M})$. These calculations should be compared with those in [5, page 246]. This completes the proof of Theorem 1.

It is worth pointing out that the formula (5) can also be used to show that $\gamma(\mathbf{b})$ is a compact subset of $\mathbf{R}^{n}$. Indeed, to see that $\gamma(\mathbf{b})$ is bounded, fix an element $m \in \mathfrak{M}$. Then $\hat{b}_{j}(m) \in \sigma_{\mathfrak{B}}\left(b_{j}\right)$ and so $\left|\hat{b}_{j}(m)\right| \leq r\left(b_{j}\right), 1 \leq j \leq n$, where

$$
r(b)=\sup \left\{|\lambda|: \lambda \in \sigma_{\mathfrak{B}}(b)\right\} \leq\|b\|
$$

denotes the spectral radius of any $b \in \mathfrak{B}$. Accordingly, if $\mathbf{u}$ is an element of $Z(\hat{\mathbf{b}}, m)$ (see (6)), then in the notation of (7.1) and (7.2) we have

$$
\|\mathbf{u}\|_{2} \leq\|\mathbf{a}(m)\|_{2}+\|\mathbf{c}(m)\|_{2} \leq\left(2 \sum_{j=1}^{n}\left|\hat{b}_{j}(m)\right|^{2}\right)^{1 / 2} \leq\left(2 \sum_{j=1}^{n} r\left(b_{j}\right)^{2}\right)^{1 / 2} .
$$

It follows that $Z(\hat{\mathbf{b}}, m)$ is contained in the ball in $\mathbf{R}^{n}$ centred at zero with radius

$$
r(\mathbf{b})=\left(2 \sum_{j=1}^{n} r\left(b_{j}\right)^{2}\right)^{1 / 2}
$$

Since this is valid for every $m \in \mathfrak{M}$, the set $\gamma(\mathbf{b})$ is also contained in this ball; see (5). This should be compared with [3, Theorem 4.1(b)] where it is shown that $\gamma(\mathbf{T})$ is contained in a ball centred at zero with radius $n^{1 / 2}\|\mathbf{T}\|$. Here $\|T\|$ is a norm satisfying [3, page 423$]$

$$
\max \left\{\left\|T_{j}\right\|: 1 \leq j \leq n\right\} \leq\|\mathbf{T}\| \leq \sum_{j=1}^{n}\left\|T_{j}\right\|
$$

shich can be associated with $\mathbf{T}$ by identifying $\mathbf{T}$ with the operator $T=$ $\sum_{j=1}^{n} T_{j} e_{j}$ acting in the Banach module $X_{(n)}$ defined over the (real) Clifford algebra $R_{(n)}$ as in [3, Section 3]. In general, $\|T\|$ is difficult to compute and so in practice the most useful statement would be that $\gamma(\mathbf{T})$ is contained in the ball centred at zero with radius $n^{1 / 2} \sum_{j=1}^{n}\left\|T_{j}\right\|$. Noting that

$$
\left.r(\mathbf{T})=\left(2 \sum_{j=1}^{n} r\left(T_{j}\right)^{2}\right)^{1 / 2} \leq\left(2 \sum_{j=1}^{n}\left\|T_{j}\right\|^{2}\right)\right)^{1 / 2} \leq 2^{1 / 2} \sum_{j=1}^{n}\left\|T_{j}\right\|
$$

we can improve this statement; it suffices to use a ball of radius $r(\mathbf{T})$. To see that $\gamma(\mathbf{b})$ is a closed set, let $\left\{\mathbf{u}^{(k)}\right\} \subseteq \gamma(\mathbf{b})$ be a sequence which is convergent to $\mathbf{u} \in \mathbf{R}^{n}$. By (5) there exist elements $m_{k} \in \mathfrak{M}$ such that

$$
\sum_{j=1}^{n}\left(\hat{b}_{j}\left(m_{k}\right)-u_{j}^{(k)}\right)^{2}=0, \quad k=1,2, \ldots
$$


The compactness of $\mathfrak{M}$ guarantees the existence of a point $m \in \mathfrak{M}$ and a subnet $\left\{m_{\alpha}\right\}$ of $\left\{m_{k}\right\}$ such that $m_{\alpha} \rightarrow m$ in $\mathfrak{M}$. This induces a subnet $\left\{\mathbf{u}^{(\alpha)}\right\}$ of $\left\{\mathbf{u}^{(k)}\right\}$ and hence, $\mathbf{u}^{(\alpha)} \rightarrow \mathbf{u}$ in $\mathbf{R}^{n}$. In particular, for each $j=1, \ldots, n$, we have $\lim _{\alpha} u_{j}^{(\alpha)}=u_{j}$ and, by continuity of $\hat{b}_{j}$, also $\lim _{\alpha} \hat{b}_{j}\left(m_{\alpha}\right)=\hat{b}_{j}(m)$. It follows from (8) that

$$
\sum_{j=1}^{n}\left(\hat{b}_{j}(m)-u_{j}\right)^{2}=\lim _{\alpha} \sum_{j=1}^{n}\left(\hat{b}_{j}\left(m_{\alpha}\right)-u_{j}^{(\alpha)}\right)^{2}=0
$$

and hence $\mathbf{u} \in \gamma(\mathbf{b})$.

It is worth summarizing and specializing our Banach algebra results to the original setting of operators on a Banach space. Recall that $\{\mathrm{T}\}^{c c}$ denotes the bicommutant of $\left\{T_{j}: 1 \leq j \leq n\right\}$. Let $\mathfrak{M}(\mathbf{T})$ denote the maximal ideal space of $\{\mathbf{T}\}^{c c}$.

Theorem 2. Let $X$ be a Banach space, $n \geq 2$ be an integer and $\mathbf{T}=$ $\left(T_{1}, \ldots, T_{n}\right)$ be a commuting $n$-tuple of elements from $L(X)$. Then

$$
\gamma(\mathbf{T})=\left\{\mathbf{u} \in \mathbf{R}^{n}: 0 \leq \sigma_{\{\mathbf{T}\}}\left(\sum_{j=1}^{n}\left(T_{j}-u_{j} I\right)^{2}\right)\right\}
$$

is a non-empty, compact subset of $\mathbf{R}^{n}$ which is contained in the ball centred at zero with radius $r(\mathbf{T})=\left(2 \sum_{j=1}^{n} r\left(T_{j}\right)^{2}\right)^{1 / 2}$. Furthermore,

$$
\gamma(\mathbf{T})=\bigcup_{m \in \mathfrak{M}(\mathbf{T})}\left\{\mathbf{u} \in \mathbf{R}^{n}: \sum_{j=1}^{n}\left(\hat{T}_{j}(m)-u_{j}\right)^{2}=0\right\} .
$$

We conclude with some remarks about the cardinality of $\gamma(\mathbf{T})$.

(I) If $\sigma\left(T_{j}\right) \subseteq \mathbf{R}$, for every $j=1,2, \ldots, n$, then we have, in the notation of Theorem 2 (see (2) and (4) with $\mathfrak{B}=\{\mathrm{T}\}^{c c}$ ), that

$$
\hat{T}_{j}(m) \in \sigma_{\{\mathbf{T}\} c}\left(T_{j}\right)=\sigma_{L(X)}\left(T_{j}\right) \subseteq \mathbf{R}, \quad 1 \leq j \leq n,
$$

for every $m \in \mathfrak{M}(\mathbf{T})$. Accordingly, the only solution in $\mathbf{R}^{n}$ of the equation $\sum_{j=1}^{n}\left(\hat{T}_{j}(m)-u_{j}\right)^{2}=0$ is $\mathbf{u}=\mathbf{T}(m)$. It follows from (9) that

$$
\gamma(\mathbf{T})=\left\{\left(\hat{T}_{1}(m), \ldots, \hat{T}_{n}(m)\right) ; m \in \mathfrak{M}(\mathbf{T})\right\}
$$

whenever all operators $T_{j}, 1 \leq j \leq n$, have real spectrum; see also [4, Theorem 1(iii)]. Since $\gamma(S)=\sigma(S) \cap R$, for every $S \in L(X)$, we have, in particular, that

$$
\gamma(\mathbf{T}) \subseteq \gamma\left(T_{1}\right) \times \cdots \times \gamma\left(T_{n}\right)
$$

see also [3, Corollary 7.4]. 
(II) If $n \geq 3$ and, for some $j_{0} \in\{1, \ldots, n\}$ the set $\sigma\left(T_{j_{0}}\right)$ contains a point from $\mathbb{C} \backslash \mathbf{R}$, then $\gamma(\mathbf{T})$ is an uncountable set. Indeed, in this case there exists $m_{0} \in \mathfrak{M}(\mathbf{T})$ such that $\hat{T}_{j_{0}}\left(m_{0}\right) \in \mathbb{C} \backslash \mathbf{R}$ and hence, if we determine the set

$$
Z\left(\hat{\mathbf{T}}, m_{0}\right)=\left\{\mathbf{u} \in \mathbf{R}^{n}: \sum_{j=1}^{n}\left(\hat{T}_{j}\left(m_{0}\right)-u_{j}\right)^{2}=0\right\}
$$

by solving the corresponding equations (7.1) and (7.2), then it is clear that the sphere and the hyperplane in $\mathbf{R}^{n}$ so specified are not degenerate (that is, they are $(n-1)$-dimensional). It is then clear from (9) that $\gamma(\mathbf{T})$ is uncountably infinite. Surprisingly, perhaps, even if $X$ is a finite dimensional space, the set $\gamma(\mathbf{T})$ is "very large" as soon as at least one of the operators $\left\{T_{j}\right\}$ does not have real spectrum $(n \geq 3)$.

(III) The situation with two commuting operators is quite different. First we note that, unlike for $n \geq 3$, it can happen that $\gamma(\mathbf{T})$ is finite even if at least one of the operators $T_{1}$ or $T_{2}$, has complex points in its spectrum. Indeed, for each $m \in \mathfrak{M}(\mathbf{T})$, it is clear that

$$
\left\{\mathbf{u} \in \mathbf{R}^{2}:\left(\hat{T}_{1}(m)-u_{1}\right)^{2}+\left(\hat{T}_{2}(m)-u_{2}\right)^{2}=0\right\}
$$

consists of at most 2 elements. Accordingly, if $\sigma\left(T_{j}\right)$ is a finite set, with $k_{j}$ elements, say, then it follows from (9) and the fact that $\hat{T}_{j}(m) \in \sigma\left(T_{j}\right)$, for every $m \in \mathfrak{M}(\mathbf{T})$, that $\gamma(\mathbf{T})$ is also a finite set with at most $2^{k_{1} k_{2}}$ elements. We note that with $T_{1}=T_{2}=I$ the number of elements in $\gamma(T)=\{(1,1)\}$ is less than the maximum number possible, namely $2\left(k_{1}=k_{2}=1\right)$. If we take $T_{1}=I$ and $T_{2}=i I$, then $\gamma(\mathbf{T})$ has 2 elements and the maximum is obtained. So, in finite dimensional spaces it is the case that $\gamma(\mathbf{T})$ is always a finite set (when $n=2$ ). For infinite dimensional spaces this need not be so. For example, if $X=l^{2}, T_{1}=I$ and $T_{2}$ is the operator with diagonal matrix $\{i / n: n=1,2, \ldots\}$, then $\gamma(\mathbf{T})$ equals

$$
\{(1,0)\} \cup\left\{\left(1 \pm n^{-1}, 0\right): n=1,2, \ldots\right\} .
$$

Accordingly, $\gamma(\mathbf{T})$ is infinite and countable. It can also happen that $\gamma(\mathbf{T})$ is infinite and uncountable. Just take $X=l^{2}, T_{1}=I$ and $T_{2}$ the operator with diagonal matrix $\left\{i r_{n} ; n=1,2, \ldots\right\}$ where $\left\{r_{n}\right\}$ is dense in $[0,1]$, say.

(IV) The inclusion (10) is not valid for operators $\left\{T_{j}\right\}$ without real spectra. Indeed, take $T_{1}=T_{2}=I$ and let $T_{3}$ be any operator such that $\sigma\left(T_{3}\right) \cap R$ is finite and $\sigma\left(T_{3}\right) \cap(\mathbf{C} \backslash \mathbf{R})$ is non-empty. Then $\gamma(T)$ is infinite by Remark (II), but

$$
\gamma\left(T_{1}\right) \times \gamma\left(T_{2}\right) \times \gamma\left(T_{3}\right)=\{1\} \times\{1\} \times\left(\sigma\left(T_{3}\right) \cap \mathbf{R}\right)
$$

is a finite set. 


\section{References}

[1] A. McIntosh and A. Pryde, 'The solution of systems of operator equations using Clifford algebras', Proc. Centre Math. Anal., vol. 9, pp. 212-222 (Australian National University, Canberra, 1985).

[2] A. McIntosh, 'Clifford algebras and applications in analysis', Lectures at the University of N.S.W. University of Sydney, joint analysis seminar, 1985.

[3] A. Mclntosh and A. Pryde, 'A functional calculus for several commuting operators', Indiana Univ. Math. J. 36 (1987), 421-439.

[4] A. McIntosh, A. Pryde and W. J. Ricker, 'Comparison of joint spectra for certain classes of commuting operators', Studia Math. 88 (1988), 23-36.

[5] W. J. Ricker, "Spectral subsets" of $\mathbf{R}^{m}$ assoiciated with commuting families of linear operators', North-Holland Math. Studies 150 (1988), 243-247.

School of Mathematics

University of New South Wales

P.O. Box 1

Kensington, N.S.W.

Australia
Department of Mathematics University of South Carolina Columbia, South Carolina U.S.A. 